\title{
Integración de los grupos de autoayuda para familiares en un programa público de tratamiento del alcoholismo
}

\section{Integration of self-guidance groups for relatives in a public program of alcoholism treatment}

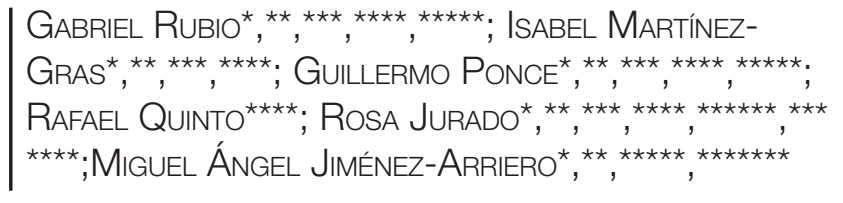

Gabriel Rubio*, ${ }^{* *},{ }^{* \star},{ }^{* \star * *},{ }^{* * \star * *}$; ISABel Martínez-

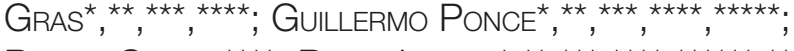

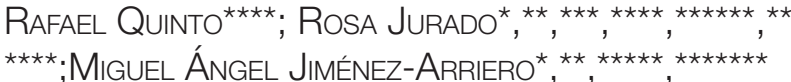

*Instituto de Investigación 12 de Octubre. Hospital Universitario 12 de octubre **Departamento de Psiquiatría. Facultad de Medicina. Universidad Complutense de Madrid.

${ }^{\star \star \star}$ Red de Trastornos Adictivos (RTA)

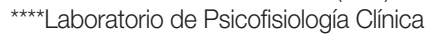

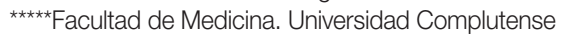

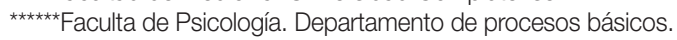

${ }^{\star \star \star \star \star \star \star}$ Centro de Investigación Biomédica en Red de salud mental (CIBERSAM)

Enviar correspondencia a:

Gabriel Rubio

Servicio de Psiquiatría. Edificio de Actividades Ambulatorias,

Planta 3, Módulo C. Hospital Universitario 12 de Octubre

Avda de Málaga, s/n

28041 Madrid.

Correo electrónico: grubio.hdoc@salud.madrid.org

\section{Resumen}

Las intervenciones con familiares de pacientes dependientes del alcohol han demostrado su eficacia para mejorar el pronóstico de la dependencia alcohólica. En la Comunidad de Madrid, la Federación de exalcohólicos ha creado la Red de centros de alternativas psicosociales a las adicciones (Red CAPA) en cuyos dispositivos se atiende a los dependientes del alcohol y a sus familiares. El objetivo de este estudio ha sido el de demostrar que la integración de terapias familiares mejoraba la salud psicológica de los familiares y el pronóstico del tratamiento de los pacientes dependientes. Fueron seleccionados 188 pacientes de nuestro programa del tratamiento del Hospital 12 de Octubre con sus respectivos familiares y se aleatorizaron en dos grupos. Un grupo A $(n=94)$ recibia el tratamiento habitual para pacientes y familiares de nuestro programa de tratamiento, mientras que en un grupo $B(n=94)$ los pacientes eran atendidos en nuestro programa de tratamiento pero sus familiares acudian a un grupo de familias de la Red CAPA. Al cabo de 12 semanas los familiares del grupo B tenían mejor salud psicológica y los pacientes tuvieron menos dias de consumo y menores tasas de abandonos del programa de tratamiento que los del grupo A. Los grupos para familiares de la Red CAPA son útiles en mejorar la salud psicológica de los familiares y en mejorar el pronóstico de la dependencia. La integración de estos centros dentro de los programas públicos de tratamiento del alcoholismo podria proporcionar una nueva forma de tratar el alcoholismo, basada en la colaboración de los establecimientos públicos y asociaciones de pacientes.

Palabras clave: alcoholismo, salud familiar, tratamiento de la dependencia alcohólica, terapia familiar del alcoholismo, asociaciones de autoayuda. recibido: Julio 2012 aceptado: Octubre 2012

\section{Abstract}

Interventions with relatives of alcohol-dependent patients have shown effectiveness in engaging unmotivated patients into treatment. The Federation of ex-alcoholics, in the Community of Madrid, has a network of self-help centers (Centers to Promote Psychosocial Alternatives against Addictions, CPAA) spread throughout the Madrid's region where alcohol-dependent patients and their families receive attention. The aim of this study was to demonstrate that the integration of these facilities could improve the psychological condition of the relatives and the outcome on the patients' treatment. 188 patients attending the treatment program at "Hospital 12 de Octubre" and their relatives were randomized into two groups: Group A ( $n=94)$ (Standard program for patients and relatives delivered at our facility) and Group B $(n=94)$ (where patients were treated with the standard program in our facilities and relatives attended a family group intervention at the (PAA) After 12 weeks, the CPAA conditions were significantly more effective than our standard program in improving psychological distress of relatives and in engaging patients into treatment. Patients belonging to group B had fewer drinking days during the treatment period. Our results support that psychological interventions delivered at CPAA centers are useful in improving psychological conditions of alcoholic patients' relatives than when undertaking the public alcoholism program. This intervention also improves outcome of alcoholic patients. The integration of these centers within public alcoholism treatment programs could provide a new way to treat alcoholism, based on collaboration of public facilities and patient associations.

Key Words: alcoholism, family health, alcohol dependence treatment, family therapy alcoholism, self-help device. 
os trastornos por el uso de alcohol representan una de las patologías más prevalentes en España. Se estima que -entre el 2-7\% de la población española son bebedores de riesgo (>60 g/etanol al día en varones) y que el $50 \%$ de dicho porcentaje son dependientes (OMS, 2011).

El beneficioso papel de la familia en el tratamiento de la dependencia alcohólica ha sido demostrado en diferentes tipos de intervenciones psicoterapéuticas. Las más contrastadas han sido las de pareja (Epstein y McCrady, 2002) y las terapias orientadas a que los familiares refuercen la abstinencia (Kirby, Marlowe y Festinger, 1999).

Con relación al primer tipo, las diferentes revisiones y meta-análisis realizados sobre este tema han demostrado que la terapia familiar mejora la adherencia terapéutica, el pronóstico de los pacientes y la participación en el seguimiento de los pacientes, cuando se comparan con los abordajes individuales (Berglung, Thelander y Jonson, 2003; Edwards y Steinglass, 1995; O'Farrell y Fals-Steward, 2001; Ripley, Cunion y Noble, 2006; Stanton y Shadish, 1997). En el meta-análisis de Stanton y Shadish (1997) se incluyeron 1571 casos que agrupaban a unos 3500 sujetos entre pacientes y familiares. Los autores concluian que las diferentes técnicas de terapia de familia eran sustancialmente mejores que las terapias de tipo individual. Dentro de los distintos abordajes familiares, la terapia de pareja, de orientación cognitivo conductual ha sido la más contratada (Epstein y McCrady, 2002), aunque lamentablemente no se encuentra disponible en todos los programas públicos de tratamiento (Fals-Stewart y Birchler, 2002).

En el alcoholismo, al igual que en otras enfermedades crónicas, el hecho de involucrar a las familias en el tratamiento mejora la adherencia terapéutica y refuerza la abstinencia de los pacientes. Las intervenciones realizadas en terapias como las de Al-Anon promueven la idea de que el paciente ha de tocar fondo para que tome conciencia de su enfermedad así como la incapacidad del familiar para ejercer un cierto control sobre el consumo de alcohol del paciente alcohólico (AIAnon Family Groups, 1990). Las técnicas basadas en que la familia sea capaz de confrontar al paciente para que acuda a tratamiento (Jonson, 1986) pueden servir para resolver los diferentes problemas que ocurren en el seno familiar, pero tienen escasa repercusión en la conducta de ingesta alcohólica del familiar dependiente y no suelen tener demasiada aceptación por parte de los familiares Barber JG, Gilbertson R (1996) (Barber y Gilbertson, 1996; Dittrich y Trapold, 1984; Loneck, Garrett, y Banks, 1996). En los últimos años se han llevado a cabo varios estudios basados en el refuerzo comunitario y en el entrenamiento de los familiares de dependientes de drogas (Community Reinforcement and family Training, CRAFT) (Kirby et al., 1999; Meyers, Miller y Smith 2001, 2002). Este tipo de abordajes, aunque poco conocidos, consisten en 12-14 sesiones a las que acuden los familiares de los pacientes en tratamiento y en las que se les enseña principalmente a reforzar las conductas de abstinencia y a que sean capaces de manejar las situaciones en las que los pacientes son expuestos a las consecuencias negativas de su consumo. Los resultados de este tipo de intervenciones duplican la capacidad de retención de los pacientes en los programas de tratamiento respecto de las intervenciones familiares realizadas bajo la filosofía de los 12 pasos (Al-Anon) (Meyer et al., 2001, 2002).

En resumen, la mayor parte de autores coinciden en señalar que un programa de tratamiento para dependientes del alcohol sería aquel que pudiese incluir de forma sistemática el apoyo a sus familiares (esposas y maridos de los/as pacientes) Este "desideratum" choca con la situación real de los programas de tratamiento del alcoholismo, bien los ubicados en los centros de salud mental o los que se llevan a cabo en los centros de atención a drogodependientes. A pesar de las recomendaciones realizadas desde la Unión Europea (Informe para la UE, 1999), en la mayor parte de estos programas la atención se centra casi exclusivamente en el paciente, relegando la atención a familiares cuando es detectado algún problema de salud mental entre ellos.

Esta situación podria paliarse de dos formas: a) que las administraciones públicas realizaran un esfuerzo para incluir estas técnicas en su programas de tratamiento de la dependencia por el alcohol; b) que las intervenciones grupales llevadas a cabo por algunas asociaciones de exalcohólicos fuesen integradas dentro de los programas públicos de tratamiento, siempre que demostrasen su eficacia.

Para dilucidar esta última cuestión nuestro grupo ha llevado a cabo este estudio con la intención de demostrar si la integración de las terapias grupales para familiares realizadas en una asociación de exalcohólicos de la Comunidad de Madrid podría mejorar la eficacia de nuestro programa de tratamiento. Los dos objetivos principales del proyecto eran: a) demostrar que los grupos de terapia familiar realizados en una asociación de exalcohólicos era más eficaz que nuestra intervención habitual en mejorar la salud psicológica de los familiares; b) los pacientes dependientes del alcohol de los familiares que acuden a la terapia de la asociación tendrán también mejor pronóstico que los pacientes que reciben el tratamiento habitual. Las variables elegidas para evaluar estos objetivos fueron la salud psicológica de los familiares (puntuaciones en escalas de salud físico-psicológica), las tasas de abandono y de recaídas de los pacientes dependientes del alcohol atendidos en nuestro centro.

En la Comunidad de Madrid, la Federación de exalcohólicos (FACOMA), ha organizado la atención a pacientes y a familiares en la denominada Red de centros de alternativas psicosociales a las adicciones (Red CAPA). Esta Red se haya diseminada por toda nuestra Comunidad y constituye un tupido tejido de centros de apoyo al tratamiento en los dispositivos de la red pública sanitaria. En estos centros se llevan a cabo tareas de asesoramiento a pacientes y familiares con problemas por el alcohol, se realizan grupos de autoayuda orientados a la abstinencia, así como intervenciones familiares en formato grupal. Este tipo de intervenciones se llevan a cabo entre 5-7 veces por semana (según los centros). Además promueven distintas actividades psicosociales como complemento de sus programas de tratamiento que tienen como finalidad afianzar más la rehabilitación del paciente dependiente del alcohol. 


\section{Método}

\section{Muestra}

La muestra fue seleccionada de los pacientes que acudieron a la Unidad de Conductas adictivas del Hospital 12 de Octubre durante un periodo de 9 meses (ver Figura 1). Fueron atendidos 310 pacientes de los que 122 renunciaron a participar por lo que fueron aleatorizados $188(\mathrm{n}=94$ pacientes para cada grupo con sus respectivos familiares). La causa más frecuente de renuncia era la negativa por parte del paciente, habitualmente varón, a que su familia acudiese a este tipo de tratamiento $(n=74)$. Hubieron 34 familiares que no deseaban participar porque las relaciones familiares estaban bastante deterioradas y en un pequeño número de casos $(n=14)$ las agendas de los familiares eran incompatibles con su asistencia a las terapias grupales. Durante el periodo de tratamiento abandonaron 51 pacientes/familiares, de forma que finalizaron el estudio 137 pacientes con sus respectivos familiares (Figura 1).

\section{Diseño}

Se trata de un estudio aleatorizado donde se comparaba el tipo de abordaje que tradicionalmente se prestaba a las familias de pacientes con problemas por alcohol (grupo A) frente a un abordaje experimental consistente en derivar a las familias a los grupos de familiares de una asociación de exalcólicos ubicada en el área de influencia del Hospital 12 de Octubre (Grupo B).

Los pacientes y sus respectivos familiares (esposa/o, madre-padre, hermanos/as e hijos) fueron aleatorizados de forma que paciente y familiar estaban integrados en el mismo grupo de tratamiento (la aleatorización se llevó a cabo mediante una tabla de números aleatorios) y después de haber firmado el consentimiento informado entraron en el estudio. Con la finalidad de evitar el sesgo evaluador de los clínicos que realizan los tratamientos, un evaluador ciego al proceso de aleatorización realizó las entrevistas basales a los familiares (RG). La misma evaluación se llevó a cabo al final del periodo de tratamiento (12 semanas). También los pacientes fueron entrevistados basalmente y tras 3 meses de tratamiento, recogiendo las variables relacionadas con el consumo y con la permanencia o no en el programa de tratamiento.

\section{Grupos de tratamiento}

Grupo A: Los pacientes con dependencia fueron atendidos en el Programa de tratamiento del alcoholismo del Hospital Universitario 12 de 0 ctubre, consistente inicialmente en sesiones grupales (1 por semana durante 12 semanas) orientadas a la prevención de recaídas (Alamo, López-Muñoz y Rubio, 2008). Sus familiares, generalmente esposas, recibieron el tipo de atención que habitualmente se les presta: explicación del programa de tratamiento; disponibilidad para atenderlos, si lo consideraban oportuno, en cualquier momento del tratamiento; evaluación psiquiátrica en el caso de que lo solicitasen. Los familiares acudieron acompañando al paciente entre 1-3 veces durante el periodo de estudio.
Grupo B: Los pacientes dependientes del alcohol recibieron el mismo tratamiento que los del grupo A, Programa de Prevención de recaídas (Alamo et al., 2008). Los familiares recibieron apoyo psicológico en los "Grupos de autoayuda para familiares" cuyas intervenciones se centraban en reforzar la abstinencia, favorecer la asistencia al tratamiento y manejar la situaciones problemáticas que ocurren durante el proceso terapéutico) (Tabla 1). Esta intervención se realizará en el centro de la Red CAPA (Asociación de exalcohólicos de Villaverde) que habitualmente colabora con nuestro programa. A este grupo de terapia acudía semanalmente un miembro del equipo (GR) y 2-3 mujeres de la asociación de exalcohólicos. Las intervenciones se realizaban en forma de terapia de grupo (1 sesión de 2 horas de duración por semana en horario de 19 a 21 horas) donde asistían entre 7-10 familiares por grupo. En cada sesión se informaba-explicaba el asunto a tratar y después los familiares exponian sus opiniones y experiencias relacionadas con el tema. El moderador aprovechaba las intervenciones de los familiares para reforzar las conductas que propiciaban los cambios implicados en la detección de recaídas y en una mejora de la capacidad de afrontamiento de las situaciones conflictivas ocurridas en el ámbito familiar. Una vez finalizadas las diez sesiones, los familiares eran invitados a continuar asistiendo a las terapias familiares en su formato habitual (grupos abiertos con una frecuencia de tres veces por semana).

Tabla 1. Aspectos que se abordaron en las terapias con los familiares en cada una de las sesiones durante el periodo de tratamiento

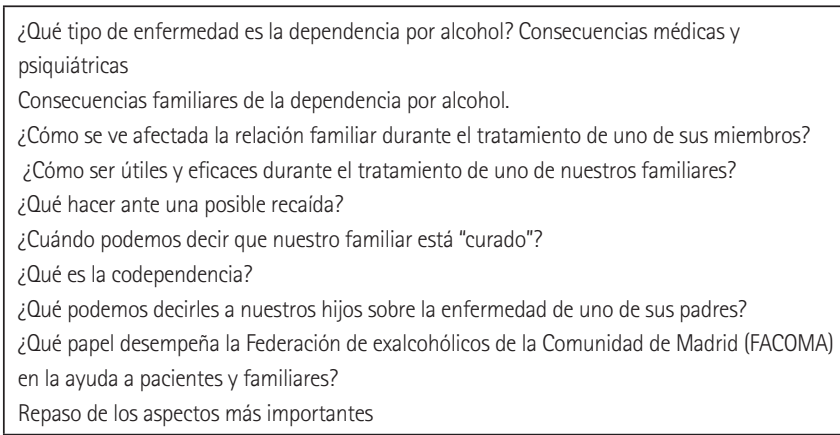

CRIBAJE

Pacientes atendidos $(\mathrm{N}=310)$

SELECCIÓN

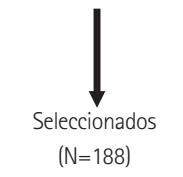




\section{Instrumentos de evaluación utilizados}

\section{Evaluación de los familiares}

Cuestionario SF-36 sobre el estado de salud: Es un cuestionario autoaplicado de 36 ítems que determina el estado de salud física y mental. Los 36 items del instrumento cubren las siguientes escalas: Función física, Rol físico, Dolor corporal, Salud general, Vitalidad, Función social, Rol emocional y Salud mental (Alonso, Prieto y Anto, 1995).

Cuestionario de 90 sintomas (Symptom Check List - 90; SCL90-R): Es un cuestionario autoaplicado diseñado en la Universidad Johns Hopkins para la evaluación de diferentes dimensiones psicopatológicas presentes en población general, en enfermos con patologías mentales o médicas y que incluye las siguientes subescalas: Somatización, Obsesión, Compulsión, Hipersensibilidad, Depresión, Ansiedad, Hostilidad, Ansiedad fóbica, Ideación Paranoide y Psicoticismo (De las Cuevas, Gonzalez de Rivera, Benitez, Monterrey, Rodriguez-Pulido y Gracia Marco, 1991).

\section{Evaluación de los pacientes}

Los pacientes fueron entrevistados y diagnosticados según criterios del manual diagnóstico y estadístico de los trastornos mentales (DSM-IV-TR) (APA 2000).

La gravedad de la dependencia se evaluó mediante la Escala de la Intensidad de la Dependencia alcohólica (EIDA) (Rubio, Urosa y Santo-Domingo, 1998). Se trata de un instrumento diseñado para recoger la gravedad del síndrome de dependencia alcohólica. Evaluación del patrón de consumo de alcohol previo al ingreso en el estudio (Sobell, Sobell y Leo, 1998). Se trata de una entrevista diseñada para determinar el patrón de consumo previo al tratamiento y durante el seguimiento.

Como marcador indirecto de consumo de alcohol se utilizó el nivel de gamma-glutamil-transferasa (GGT) basal y a las 12 semanas de tratamiento.

\section{Análisis estadístico}

Basalmente las variables continuas fueron comparadas mediante la prueba de la T de Student y las cualitativas con la Chi-cuadrado. Para analizar las diferencias en la salud psicológica de los familiares incluidos en los dos grupos de tratamiento se utilizó la prueba de la T de Student comparar las pruebas psicométricas y otros valores de los familiares y de los pacientes de ambos grupos tanto basal como tras las 12 semanas de tratamiento.

\section{Resultados}

\section{Grupo de familiares}

La muestra de familiares estaba formada por 157 esposas, 10 maridos, 13 madres, 6 hijos y 2 padres, distribuidos de forma similar entre los dos grupos. No se evidenciaron diferencias en las puntuaciones basales de las escalas que evaluaban síntomas psicológicos y estado de salud (ver tabla 2). A lo largo del seguimiento abandonaron de forma significativa más familiares del grupo A que del B (32 vs 19). En el grupo A, la recaída del paciente conllevó el abandono del seguimiento por parte del familiar. Los motivos más frecuentes para el abandono en el grupo B fueron la aparición de problemas de agenda que les dificultaba acudir a los grupos en el horario pactado $(n=5)$ y la recaída del paciente $(n=14)$. Todos los integrantes del grupo $B$ acudieron a más de 7 sesiones a lo largo del estudio (Tabla 2).

Finalizado el estudio, los familiares que habian acudido a las terapias para familiares (Grupo B) reconocian haber recibido una información que antes desconocian sobre la dependencia por alcohol. Psicológicamente los integrantes del grupo B, respecto de los del otro grupo, habían disminuido de forma significativa sus puntuaciones en las subescalas de somatización, depresión, ansiedad y fobia de la SCL-90R (Tabla 2). Respecto del estado de salud, los familiares del grupo B habían mejorado de forma significativa en las siguientes subescalas de la SF-36: limitaciones para el rol social, salud mental y limitación por problemas emocionales (Tabla 3).

Cabía la posibilidad de que los familiares con peores puntuaciones en salud psicológica fuesen los que hubieran abandonado, bien en el grupo A o en el B. Para comprobar este hecho se compararon las puntuaciones basales de los abandonos de ambos grupos y no encontramos diferencias significativas en ninguna de las subescalas (en todos los casos p >0.1).

\section{Grupo de pacientes}

Basalmente no había diferencias en el patrón de consumo ni en el patrón de complicaciones entre los pacientes de ambos grupos (Tabla 4). A lo largo del seguimiento abandonaron significativamente más pacientes en el grupo A que en el B (32 vs 19). Al final del estudio los pacientes incluidos en el grupo $B$ tuvieron de forma significativa menos días de consumo y menores niveles de GGT que los del grupo A, lo que era indicativo de que habian consumido menos cantidad de alcohol.

También en este caso comparamos las variables basales relacionadas con el consumo de alcohol y con la dependencia de los abandonos de ambos grupos y tampoco encontramos diferencias significativas entre ellos ( $p>0.2$ para las siguientes variables: unidades de bebida estándar, puntuación en le escala EIDA y niveles de GGT) (Tabla 4). 
Tabla 2. Diferencias sociodemográficas entre familiares incluidos en dos modalidades de abordaje familiar

\begin{tabular}{|c|c|c|c|c|c|c|}
\hline \multirow[t]{2}{*}{ VARIABLE } & \multicolumn{2}{|c|}{ Grupo A: tratamiento familiar habitual } & \multicolumn{2}{|c|}{$\begin{array}{c}\text { Grupo B: Tratamiento familiar en un centro } \\
\text { de la red CAPA }\end{array}$} & \multirow[t]{2}{*}{$\begin{array}{c}\text { Significación de las } \\
\text { diferencias basales entre } \\
\text { grupos }\end{array}$} & \multirow[t]{2}{*}{$\begin{array}{c}\text { Significación de las difer- } \\
\text { encias entre grupos a las } 12 \\
\text { semanas }\end{array}$} \\
\hline & $\begin{array}{l}\text { Basalmente } \\
\quad(n=94)\end{array}$ & $\begin{array}{c}12 \text { semanas } \\
(n=62)\end{array}$ & $\begin{array}{l}\text { Basalmente } \\
\quad(n=94)\end{array}$ & $\begin{array}{c}12 \text { semanas } \\
(n=75)\end{array}$ & & \\
\hline Familiares & 94 & 62 & 94 & 75 & NS & NS \\
\hline Esposas/os n, (\%) & $85(90.4)$ & $55(88.7)$ & 82 (89.3) & 65 (86.6) & NS & NS \\
\hline Otros (hermanos/padres) & $9(9.5)$ & $7(11.2)$ & $12(12.7)$ & 10 (13.3) & NS & NS \\
\hline Edad & $42.05(20.1)$ & $43.36(18.2)$ & $43.78(19.3)$ & $42.56(18.8)$ & NS & NS \\
\hline Género (mujer) n, (\%) & 86 (91.4) & 55 (88.7) & $84(89.3)$ & 66 (88.0) & NS & NS \\
\hline $\begin{array}{l}\text { Nivel educativo } \\
\text { Años de escolaridad, } x_{1}(\mathrm{ds})\end{array}$ & $12.23(6.1)$ & $11.92(6.0)$ & $11.45(7.0)$ & $11.67(7.6)$ & NS & NS \\
\hline $\begin{array}{l}\text { Actividad profesional } \\
\text { Ama de casa } n_{1}(\%)\end{array}$ & 75 (79.7) & $49(79.0)$ & $69(73.4)$ & 57 (76.0) & NS & NS \\
\hline
\end{tabular}

Tabla 3. Puntuaciones en las escalas de salud física y psicológica de los familiares de pacientes con dependencia por alcohol

\begin{tabular}{|c|c|c|c|c|c|c|}
\hline \multirow[t]{2}{*}{ VARIABLE } & \multicolumn{2}{|c|}{ Grupo A: tratamiento familiar habitual } & \multicolumn{2}{|c|}{$\begin{array}{l}\text { Grupo B: Tratamiento familiar en un } \\
\text { centro de la red CAPA }\end{array}$} & \multirow{2}{*}{$\begin{array}{c}\text { Significación de las } \\
\text { diferencias basales } \\
\text { entre grupos }\end{array}$} & \multirow{2}{*}{$\begin{array}{l}\text { Significación de las } \\
\text { diferencias entre gru- } \\
\text { pos a las } 12 \text { semanas }\end{array}$} \\
\hline & $\begin{array}{l}\text { Basalmente } \\
\qquad(n=94)\end{array}$ & $\begin{array}{l}12 \text { semanas } \\
\quad(n=62)\end{array}$ & $\begin{array}{l}\text { Basalmente } \\
\qquad(n=94)\end{array}$ & $\begin{array}{c}12 \text { semanas } \\
(\mathrm{n}=75)\end{array}$ & & \\
\hline \multicolumn{7}{|l|}{ SCL-90R } \\
\hline $\begin{array}{l}\text { Somatización, } x_{1}(d s) \\
\text { Obsesión-compulsión, } x_{1}(d s) \\
\text { Sensibilidad interpersonal, } x_{1}(d s) \\
\text { Depresión, } x_{1}(d s) \\
\text { Ansiedad, } x_{1}(d s) \\
\text { Hostilidad, } x_{1}(d s) \\
\text { Fobia, } x_{1}(d s) \\
\text { Ideación paranoide, } x_{1}(d s) \\
\text { Psicoticismo, } x_{1}(d s)\end{array}$ & $\begin{array}{l}0.87(0.60) \\
0.62(0.50) \\
0.50(0.35) \\
1.83(0.60) \\
1.64(0.42) \\
0.44(0.32) \\
1.30(0.25) \\
0.37(0.39) \\
0.18(0.21)\end{array}$ & $\begin{array}{l}0.80(0.62) \\
0.60(0.53) \\
0.48(0.37) \\
1.60(0.63) \\
1.44(0.48) \\
0.42(0.35) \\
1.27(0.28) \\
0.34(0.37) \\
0.19(0.23)\end{array}$ & $\begin{array}{l}0.90(0.61) \\
0.56(0.52) \\
0.48(0.39) \\
1.88(0.66) \\
1.68(0.49) \\
0.41(0.30) \\
1.32(0.27) \\
0.34(0.36) \\
0.16(0.22)\end{array}$ & $\begin{array}{l}0.59(0.61) \\
0.52(0.50) \\
0.40(0.35) \\
0.90(0.67)^{*} \\
1.10(0.51)^{*} \\
0.44(0.32) \\
0.90(0.35)^{*} \\
0.33(0.38) \\
0.14(0.20)\end{array}$ & $\begin{array}{l}\text { NS } \\
\text { NS } \\
\text { NS } \\
\text { NS } \\
\text { NS } \\
\text { NS } \\
\text { NS } \\
\text { NS } \\
\text { NS }\end{array}$ & $\begin{aligned} t=1.99, g l & =135, p<0.05 \\
& N S \\
& N S \\
t=6.25, g & =135, p<0.01 \\
t=3.98, g l & =135, p<0.01 \\
& N S \\
t=6.73, g & =135, p<0.01 \\
& N S \\
& N S\end{aligned}$ \\
\hline \multicolumn{7}{|l|}{ SF-36 } \\
\hline $\begin{array}{l}\text { Funcionamiento Fisico, } x_{1}(d s) \\
\text { Limitación por problemas físicos, } \\
x_{1} \text { (ds) } \\
\text { Dolor corporal, } x_{1}(d s) \\
\text { Funcionamiento o rol social, } x_{1}(d s) \\
\text { Salud mental, } x_{1}(d s) \\
\text { Limitación por problemas } \\
\text { emocionales, } x_{1}(d s) \\
\text { Vitalidad, energia o fatiga, } x_{1}(d s) \\
\text { Percepción general de la salud, } \\
x_{1} \text { (ds) }\end{array}$ & $\begin{array}{c}92.45(11.61) \\
90.23(22.34) \\
87.32(25.85) \\
78.01(12.43) \\
60.73(12.32) 82.31 \\
(21.12) \\
60.56(13.63) 72.76 \\
(15.78)\end{array}$ & $\begin{array}{l}93.02(10.74) \\
91.11(21.33) \\
88.34(24.12) \\
82.14(11.05) \\
68.44(10.80) \\
86.70(19.34)\end{array}$ & $\begin{array}{l}91.34(10.43) \\
92.14(21.33) \\
86.90(22.30) \\
77.54(13.56) \\
61.46(13.50) \\
83.12(20.32) \\
\\
61.01(14.11) \\
73.05(14.27)\end{array}$ & $\begin{array}{c}94.44(11.05) \\
91.03(18.79) \\
89.22(23.11) \\
89.75(10.33)^{*} \\
72.86(9.93)^{*} \\
93.96(18.66)^{*} \\
67.51(13.65) \\
78.71(13.96\end{array}$ & $\begin{array}{l}\text { NS } \\
\text { NS } \\
\text { NS } \\
\text { NS } \\
\text { NS } \\
\text { NS } \\
\text { NS } \\
\text { NS }\end{array}$ & $\begin{array}{c}N S \\
N S \\
N S \\
t=3.59, g l=135, p<0.01 \\
t=1.96, g l=135, p<0.05 \\
t=2.02, g l=135, p<0-05 \\
\\
\text { NS } \\
\text { NS }\end{array}$ \\
\hline
\end{tabular}

SCL-90R: Symptom Check List - 90; SCL-90-R): SF-36: Cuestionario de Salud SF-36

$\mathrm{X}=$ media, ds=desviación estándar 
Tabla 4. Diferencias clínicas de los pacientes incluidos en dos modalidades de tratamiento para familiares

\begin{tabular}{|c|c|c|c|c|c|c|}
\hline \multirow[t]{2}{*}{ Variable } & \multicolumn{2}{|c|}{$\begin{array}{c}\text { Grupo A: Pacientes y familiares recibian el } \\
\text { tratamiento }\end{array}$} & \multicolumn{2}{|c|}{$\begin{array}{l}\text { Grupo B: Los pacientes recibian el tratami- } \\
\text { ento habitual y sus familiares en un centro } \\
\text { de la red CAPA }\end{array}$} & \multirow[t]{2}{*}{$\begin{array}{l}\text { Significación de las } \\
\text { diferencias basales } \\
\text { entre grupos }\end{array}$} & \multirow[t]{2}{*}{$\begin{array}{c}\text { Significación de las } \\
\text { diferencias entre } \\
\text { grupos a las } 12 \\
\text { semanas }\end{array}$} \\
\hline & $\begin{array}{l}\text { Basalmente } \\
\qquad(n=94)\end{array}$ & $\begin{array}{l}12 \text { semanas } \\
(\mathrm{n}=62)\end{array}$ & $\begin{array}{l}\text { Basalmente } \\
\qquad(n=94)\end{array}$ & $\begin{array}{c}12 \text { semanas } \\
(\mathrm{n}=75)\end{array}$ & & \\
\hline Edad, $\times$ (ds) & $42.3(8.4)$ & $41.5(8.2)$ & $44.7(10.1)$ & $42.4(9.7)$ & NS & NS \\
\hline Género (varones), n (\%) & $80(85.1)$ & $49(79.0)$ & $82(87.2)$ & $60(80.0)$ & NS & NS \\
\hline $\begin{array}{l}\text { Nivel educativo (años de estudios), } \\
x \text { (ds) }\end{array}$ & $15.1(8.1)$ & $14.8(9.0)$ & $14.9(9.0)$ & $14.2(8.9)$ & NS & NS \\
\hline $\begin{array}{l}\text { Actividad profesional } \\
\text { Desempleo, } \mathrm{n}(\%)\end{array}$ & $51(54.2)$ & $31(50)$ & $53(56.3)$ & $37(49.3)$ & NS & NS \\
\hline $\begin{array}{l}\text { Edad de comienzo del consumo de } \\
\text { alcohol, } x \text { (ds) }\end{array}$ & $15.3(2.1)$ & $14.2(3.0)$ & $14.7(2.5)$ & $13.4(2.6)$ & NS & NS \\
\hline Años de dependencia alcohólica, x (ds) & $8.8(3.4)$ & $8.5(3.0)$ & $9.0(4.0)$ & $8.9(3.7)$ & NS & NS \\
\hline \multicolumn{7}{|l|}{ Consumo de alcohol } \\
\hline $\begin{array}{l}\text { Dias de consumo en el mes previo, } \\
x \text { (ds) }\end{array}$ & 28.4(1.2) & & $28.6(1.2)$ & & NS & \\
\hline $\begin{array}{l}\text { Unidades de bebida por dia de con- } \\
\text { sumo; } \text { (ds) }\end{array}$ & $24.1(10.3)$ & & $27.4(11.6)$ & & NS & \\
\hline $\begin{array}{l}\text { Puntuación en la Escala de Intensidad } \\
\text { de la dependencia por alcohol (EIDA), } \\
\text { x (ds) }\end{array}$ & $32.2(10.1)$ & & $31.74(9.8)$ & & NS & \\
\hline Niveles de GGT, x (ds) & $84.3(12.3)$ & $61.2(10.3)$ & $87.5(11.5)$ & $53.1(11.3)$ & NS & $\begin{array}{c}t=4.09, g l=135 \\
p<0.05\end{array}$ \\
\hline $\begin{array}{l}\text { Abandonos durante el tratamiento, } \\
\mathrm{n}(\%)\end{array}$ & & $32(34.9)$ & & $19(20.2)$ & & $X^{2}=3.87, g \mid=1, p=0.049$ \\
\hline $\begin{array}{l}\text { Dias de consumo durante el tratami- } \\
\text { ento, } x \text { (ds) }\end{array}$ & & $14.2(8.1)$ & & $9.2(6.2)$ & & $\begin{array}{c}t=4.06, g l=135, \\
p<0.001\end{array}$ \\
\hline
\end{tabular}

$X=$ media, $d s=$ desviación estándar

\section{Discusión}

Este es el primer estudio que evalúa la eficacia de las intervenciones familiares llevadas a cabo en una asociación de exalcohólicos integrada dentro de un programa de tratamiento de la dependencia por alcohol perteneciente a la red pública sanitaria. Los resultados más relevantes son que los familiares atendidos en la asociación de exalcohólicos mejoraban su estado psicológico y de salud más que los familiares que acudian periódicamente con su familiar a la consulta de seguimiento de su psiquiatra. Además, los pacientes de los familiares incluidos en el grupo de terapia familiar tuvieron menos abandonos y menos días de consumo durante le periodo de tratamiento.

Los resultados relacionados con la mejoría en la salud física y psicológica de los familiares incluidos en terapia familia coinciden con los publicados por otros autores Kahler, McCrady y Epstein, 2003; Kirby et al., 1999; Meyer et al., 2002; Oreo et al., 2007). El tipo de manifestaciones clínicas más frecuentes eran de tipo ansioso y depresivo, lo que se corresponde con una situación de alerta mantenida (Meyer et al., 2002). Las intervenciones familiares en el grupo B estaban orientadas a conocer la enfermedad y detectar las recaídas, pero en ellas los familiares contaban sus preocupaciones y hablaban de sus problemas psicológicos en relación al alcoholismo, lo que también sirvió para disminuir el grado de estrés psicológico y mejoró algunas áreas de la salud física. Además, durante las sesiones, y tomando como referencia las informaciones de las parejas de los pacientes, se detectaron también problemas psicológicos en los adolescentes de más del $40 \%$ de las familias del grupo B. Esto pone de relieve la importancia de este tipo de abordajes para detectar las complicaciones psicológicas ocurridas en parejas e hijos de pacientes con dependencia.

El hecho de que se produjeran menos abandonos entre los pacientes también ha sido señalado en otros trabajos (Kirby et al., 1999 ) y evidencia que la información y las estrategias recibidas por los familiares contribuye a la evitación de las recaídas y a su detección precoz (Meyer et al., 2002).

Por último, y probablemente lo más importante, es que la integración de las terapias de estas asociaciones en los programas de tratamiento de la red pública, es posible y es deseable. Hasta ahora, la colaboración de estos colectivos (incluido AA) con los centros de la red pública se reducía a aceptar a los pacientes y sus familiares que eran derivados desde los centros de tratamiento, o en el mejor de los casos, a la existencia de una coordinación entre el responsable del programa de alcohol con aquellas asociaciones próximas territorialmente. Sin embargo, nuestro estudio demuestra que la integración de estas asociaciones dentro de un programa público de tratamiento ofrece importantes ventajas a familiares (mejora de sus 
estado de salud psicológica y física), pacientes (mayor adherencia y menos días de consumo) y a los responsables de los programas de tratamiento (menos abandonos, posibilidades de centrar los esfuerzos técnicos en aquellos pacientes con mayor complejidad clínica; posibilidad de elegir entre diferentes centros de exalcohólicos próximo al lugar donde reside el paciente). Uno de los problemas detectados a la hora de poner en marcha este tipo de colaboración es la resistencia de los familiares a acudir a una asociación de exalcohólicos. Para evitarla, diseñamos un folleto donde se explicaba el programa terapéutico y en el que se recogía que las terapias de esa asociación formaban parte de nuestro programa terapéutico. Con el tiempo, y una vez finalizado el proyecto, algunos pacientes pedian acudir a los grupos de autoayuda de la asociación y de hecho, esos grupos solemos utilizarlos como complemento de nuestro abordaje.

Este estudio tiene claras implicaciones clínicas: si este nuevo modelo de funcionamiento se generalizase en nuestro entorno se traduciria en un aumento de los recursos asistenciales para tratar a estos pacientes y a sus familiares, es posible que los costes derivados de las recaídas también se viesen reducidos, al ser significativamente menores y por último, esta sinergia permite que los centros públicos puedan centrarse en aquellas intervenciones más complejas desde el punto de vista técnico, dejando que otras intervenciones (atención a familias y grupos de autoayuda) puedan ser realizadas en este tipo de asociaciones. Además, cuando se dan altas desde los programas de tratamiento se puede contar con estos centros para que en ellos se sigan llevando a cabo la rehabilitación de pacientes y familiares.

Una de las principales limitaciones de nuestro proyecto es que los resultados no se pueden generalizar a otros colectivos ya que desconocemos el grado de formación en entrenamiento para familiares de las asociaciones de AA o de otros grupos de exalcohólicos.

\section{Agradecimientos}

Proyecto Financiado por el Ministerio de Sanidad, Política social e igualdad en el ejercicio 2010, según acuerdo del consejo interterritorial del SNS para la distribución de fondos a las CCAA.

A la Oficina Regional de salud Mental de la Comunidad de Madrid por el apoyo a esta iniciativa. A la Federación de exalcohólicos de la Comunidad de Madrid, y en especial a su presidente, D. Antonio Gonzalez Catena por su apoyo incondicional para llevar a cabo el estudio.

\section{Conflicto de intereses}

No existe ningún conflicto de intereses.

\section{Referencias}

Al-Anon Family Groups. (1990).Una Guía para la Familia del Alcohólico. In all our affairs: Making crises work for you. New York: Author.

Alamo, C., López-Muñoz, F. y Rubio G. (Eds) (2008). Avances en Drogodependencias (pp. 465-474). Las Palmas de Gran Canaria. GAFOS.

Alonso, J., Prieto, L. y Anto, J. M. (1995). La versión española del SF-36 Health Survey (Cuestionario de Salud SF-36): un instrumento para la medida de los resultados clínicos. Medicina Clínica, 104, 771-776.

American Psychiatric Association (2000) Diagnostic and Statistical Manual of Mental Disorders. 4th ed. Text rev. American Psychiatric Association, Washington, DC.

Barber, J. G. y Gilbertson, R. (1996). An experimental study on brief unilateral intervention for the partners of heavy drinkers. Research on Social work Pratice, 6, 325-336.

Benishek, L. A., Kirby K.C. y Dugosh, K. L. (2011). Prevalence and frequency of problems of concerned family members with a substance-using loved one. American Journal of Drug and Alcohol Abuse, 37, 82-88. doi: 10.3109/00952990.2010.540276.

Berglund, M., Thelander, S. y Jonsson, E. (2003). Treating Alcohol and Drug Abuse: An Evidence Based Review (pp. 43-88). Weinheim: Wiley-VCH.

De las Cuevas, C., Gonzalez de Rivera, J. L., Benitez MH; Monterrey, A. L., Rodriguez-Pulido, F. y Gracia Marco, R. (1991). Análisis factorial de la versión española del SCL-90-R en la población general. Anales de Psiquiatría, 7, 93-96.

Dittrich, J. E. y Trapold, M. A. (1984). A treatment program for wives of alcoholics: an evaluation. Bulletin of the Society of Psychologists in Addictive Behaviors, 3, 91-102.

Edwards, M. E. y Steinglass, P. (1995). Family therapy treatment outcomes for alcoholism. The Journal of Marital and Family Therapy, 21, 475-509. doi: 10.1111/j.1752-0606.1995.tb00176.x.

Epstein, E. y McCrady, B. (2002). Couple therapy in the treatment of alcohol problems. In: Gurman AS, Jacobson NS (Eds.), Clinical handbook of couple therapy, 3rd ed. (pp. 597-628). New York: Guilford Press.

Epstein, E. y McCrady B. (1998). Behavioral couples treatment of alcohol and drug use disorders: current status and innovations. Clinical Psychology Review, 18, 689-711. doi: 10.1016/S02727358(98)00025-7

Fals-Stewart, W. y Birchler, G. R. (2001). A national survey of the use of couples therapy in substance abuse treatment. Journal of Substance Abuse Treatment, 20, 277-283. doi:10.1016/S0740-5472(01)00165-9

Informe para la Unión Europea de EUROCARE y COFACE (1999) Problemas por el Alcohol en la Familia. Madrid: Agencia Antidroga.

Johnson, V. E. (1986). Intervention: How to help those who don't want help. Minneapolis, MN: Johnson Institute.

Kahler, C. W., McCrady, B.S. y Epstein E. E. (2003). Sources of distress among women in treatment with their alcoholic partners. Journal of Substance Abuse Treatment, 24, 257-265. doi: 10.1016/S07405472(03)00033-3 
Kirby, K. C., Marlowe, D. B. y Festinger, D. S. (1999). Community reinforcement training for family and significant others of drug abusers: a unilateral intervention to increase treatment entry of drug users. Drug and Alcohol Dependence, 56, 85-96.

Loneck, B., Garrett, J., y Banks S. M. (1996). A comparison of the Johnson Intervention with four other methods of referral to outpatient treatment. American Journal of Drug and Alcohol Abuse, 22, 233246.

Meyers, R. J., Miller, W. R. y Smith, J. E. (2002). A randomized trial of two methods for engaging treatment-refusing drug users through concerned significant others. Journal of Consulting and Clinical Psychology, 70, 1182-1185. doi: 10.1037/0022-006X.70.5.1182.

Meyers, R. J., Miller, W. R. y Smith, J. E. (2001). Community reinforcement and family training (CRAFT). In: Meyers R. J., Miller W. R., eds. A community reinforcement approach to addiction treatment (pp. 147-160). New York: Cambridge University Press.

Oreo, A. y Ozgul, S. (2007). Grief experiences of parents coping with an adult child with problem substance use. Addiction Research and Theory, 15, 71-83. doi:10.1080/16066350601036169

Organización Mundial de la salud. (2011) Global status report on alcohol and health. Geneva, Switzerland: World Health Organization.

O'Farrell, T. J. y Fals-Stewart, W. (2001). Family-involved alcoholism treatment: an update. In: Galanter, M. (Ed.), Recent developments in alcoholism, vol. 15: services research in the era of managed care (pp. 329-356). New York: Brunner-Routledge.

Ripley, J., Cunion, A. y Noble, N. (2006). Alcohol abuse in marriage and family contexts: relational pathways to recovery. Alcohol Treatment Quarterly, 24, 171-184. doi: 10.1300/J020v24n01_10.

Rubio, G., Urosa, B. y Santo-Domingo, J. (1998). Validación de la escala de la intensidad de la dependencia alcohólica. Psiquiatría Biológica, 5 (supp/ 1), 44-47.

Sobell, L. C., Sobell, M. B. y Leo, G. I. (1998). Reliability of a timeline method: assessing normal drinkers' reports of recent drinking and a comparative evaluation across several populations. British Journal of Addiction, 83, 393-402.

Stanton, M. D. y Shadish, W. R. (1997). Outcome, attrition, and familycouple treatment for drug abuse: a meta-analysis and review of the controlled, comparative studies. Psycholical Bulletin, 122,170-191. 